\title{
Resonance-Induced Enhancement of the High-Order Harmonic Generation in Plasma
}

\author{
Rashid A. Ganeev* \\ Scientific Association Akadempribor, Uzbekistan Academy of Sciences, Akademgorodok, Tashkent 100125, Uzbekistan
}

\begin{abstract}
Resonance-induced enhancement of the single harmonic generating in laser-produced plasmas allows achieving the microJoule energies for the coherent pulses in the extreme ultraviolet region using the moderate-intensity lasers. We review various approaches for improving the enhancement of single harmonic, which paves the way for a growth of harmonic yield in the short-wavelength range for various applications.
\end{abstract}

Keywords: Laser plasma, high-order harmonic generation.

\section{INTRODUCTION}

A bright, monochromatic, coherent source in the extreme ultraviolet (XUV) range could be useful for many applications. Among the applications one can mention the study of the time evolution of the surface states [1], atomic and molecular spectroscopy [2-4], generation of attosecond pulses [5], interferometry and holography [6], nonlinear optics using harmonic generation [6], etc.

$\mathrm{X}$-Raylasers and high-order harmonics are the coherent sources of radiation in this region. Although the latter sources have a better coherence than the X-Ray lasers, they are not monochromatic, unless one selects some harmonic using a monochromator. The use of phase matching conditions to replace the well-known plateau of harmonic distribution by the group of intense harmonics is a step toward this direction $[7,8]$. Though the generation of intense single harmonic looks unrealistic at the moment, there are some techniques, which can considerably improve a single harmonic yield with regard to the neighboring harmonics. The possibility of the enhancement of the high-order harmonic generation (HHG) in gaseous media using the atomic and ionic resonances has extensively been studied theoretically $[9,10]$. This approach can be an alternative to the phase matching of the pump and harmonic waves using the gasfilled waveguides [11]. Furthermore, for the plasmas generated on the surfaces of some solid targets, the resonance conditions between the harmonic wavelength and the excited states of neutrals and singly charged ions can lead to enhancement of the yield for some specific harmonic orders $[12,13]$. The availability of a much wider range of target materials for plasma HHG compared to a few gases increases the possibility of the resonance of an ionic transition with a harmonic wavelength in the former case.

Recently, a considerable variation of the harmonic distribution during the HHG from indium plasma has been reported [14]. The resonance-induced enhancement was achieved by changing the harmonic wavelength, which

*Address correspondence to this author at the Scientific Association Akadempribor, Uzbekistan Academy of Sciences, Akademgorodok, Tashkent 100125, Uzbekistan; E-mail: rashid_ganeev@yahoo.com enabled the overlap of these harmonics with the ionic transitions with strong oscillator strengths. Another approach for the tuning of harmonic wavelength based on the chirp variations of driving radiation has been reported during gas HHG studies [15-17]. Moreover, the tuning of harmonics and their "sharpness" were demonstrated using a combination of the external control of laser chirp and the intensity-induced variation of laser chirp inside a nonlinear medium [16, 17].

In this mini-review, we discuss recent studies of the enhancement of a single harmonic intensity in different parts of plateau in the case of plasma HHG. After brief overview of the experimental details, we analyze the giant single harmonic generation in indium plasma and other plasmas where the harmonic enhancement was reported. We also discuss the conditions of single harmonic enhancement at strong excitation of the plasma by femtosecond radiation and using the ultraviolet laser.

\section{EXPERIMENTAL DETAILS}

The reviewed studies were carried out using the ultrashort pulses $(35,48$, and $150 \mathrm{fs})$ of the chirped-pulse amplification Ti: sapphire lasers, operating at a $10-\mathrm{Hz}$ pulse repetition rate. A portion of uncompressed radiation (pulse duration $t=200 \mathrm{ps}$ ) was split from the main beam by a beam splitter and used as a prepulse. This prepulse beam was focused by a spherical lens on the target kept in a vacuum chamber (Fig. 1). The laser intensity on the target surface was varied between $5 \times 10^{9}$ and $9 \times 10^{10} \mathrm{~W} \mathrm{~cm}^{-2}$, which was sufficient for the optimal plasma formation. The plasma length was varied in the range of $0.4-1 \mathrm{~mm}$. Various solidstate targets were used in these plasma HHG experiments. After some adjustable delay varied between 20 and $100 \mathrm{~ns}$, the ultrashort laser pulse (central wavelength $790-800 \mathrm{~nm}$, spectral width $12-35 \mathrm{~nm}$ ) was focused on the plasma area from a direction parallel to the target surface. The optimization of harmonic generation was performed by appropriate focusing of the femtosecond radiation in front of, inside, or after the plasma plume.

The intensity of driving laser pulse at the preformed plasma was adjusted between $1 \times 10^{14}$ and $4 \times 10^{15} \mathrm{~W} \mathrm{~cm}^{-2}$. The harmonics did not absorb in the plasma due to its relatively low concentration $\left(\sim 10^{17} \mathrm{~cm}^{-3}\right)$. The high-order har- 


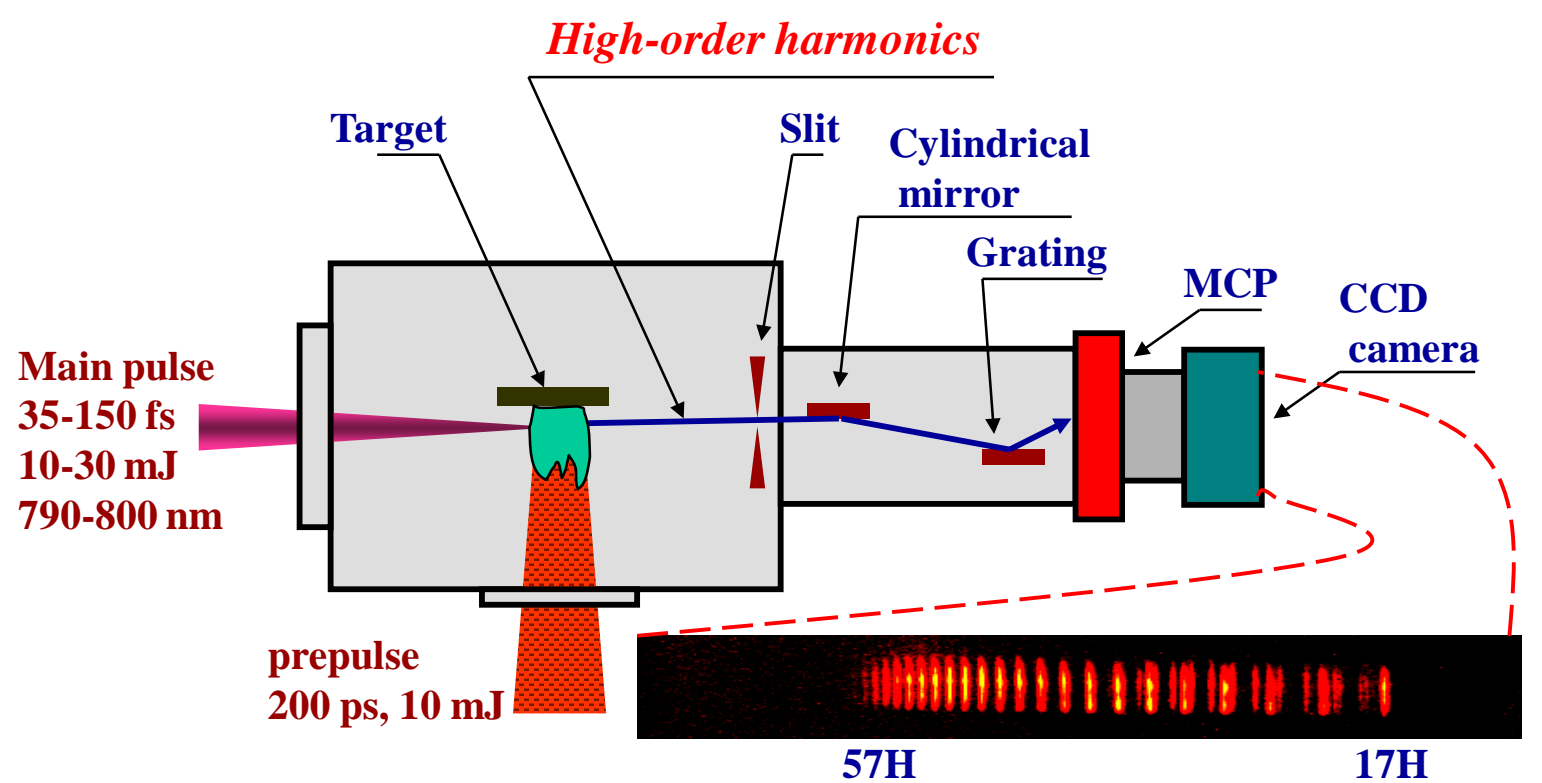

Fig. (1). A schematic view illustrating the high-order harmonic generation in laser-produced plasma.

monics generating in laser plasma were analyzed by a grazing incidence XUV spectrograph, detected by a micro channel plate with a phosphor screen, and recorded by a CCD camera. Adjusting the distance between the gratings in the compressor changed the chirp of laser pulses. Details of the experimental arrangements can be found elsewhere $[18,19]$. The absolute calibration of harmonic efficiency was performed using the technique described in Ref. [20].

\section{GIANT ENHANCEMENT OF THE $13^{\text {TH }}$ HARMONIC GENERATING IN THE INDIUM PLASMA}

First demonstration of the giant resonance-induced enhancement of the single harmonic generated in plasma was reported in Ref. [14]. A typical harmonic spectrum obtained from the In plasma using the $796 \mathrm{~nm}, 150$ fs laser pulses is shown in Fig. (2). High-order harmonics were observed in these experiments and showed a plateau pattern (Fig. 2, curve 1). The harmonic spectrum generated from silver plasma under the same experimental conditions is presented here for the comparison, which showed an analogous characteristic plateau region for the harmonics exceeding the $9^{\text {th }}$ order (Fig. 2, curve 2). The conversion efficiency at the plateau region in the case of In plasma was measured to be $8 \times 10^{-7}$. The most intriguing feature observed in these studies was a very strong $13^{\text {th }}$ harmonic, whose intensity was almost two orders of magnitude higher than those of the neighboring harmonics. The conversion efficiency of the $13^{\text {th }}$ harmonic was $8 \times 10^{-5}$, and for the pump energy of $10 \mathrm{~mJ}$, this corresponded to $0.8 \mu \mathrm{J}$ [14].

After the observation of such unusual harmonic distribution, the question arises whether the strong emission associated with $13^{\text {th }}$ harmonic $(\lambda=61.2 \mathrm{~nm})$ originates from the amplified spontaneous emission, re-excitation of plasma by a femtosecond beam, or nonlinear optical process related with the enhancement of a single harmonic due to its spectral proximity to the resonance transitions.

The pump laser polarization was varied to analyze the strong emission near $61 \mathrm{~nm}$ in the harmonic spectrum. Small deviation from linear polarization led to a considerable decrease of the $61.2 \mathrm{~nm}$ radiation intensity, which is a typical behavior for the high-order harmonics. The application of circularly polarized laser pulses led to the complete disappearance of $61.2 \mathrm{~nm}$ emission (see inset in Fig. 2). At the same time, the excited lines of plasma spectrum observed at different polarizations of main beam remained unchanged, which clearly shows that the strong $61.2 \mathrm{~nm}$ emission was generated through the nonlinear optical process.

The wavelength of main pump beam was tuned to analyze whether the excited ionic transitions from indium plasma influence the plateau pattern of harmonic distribution. The central wavelength of the output radiation of Ti: sapphire laser was tuned between 770 and $796 \mathrm{~nm}$. The $13^{\text {th }}$ harmonic output was considerably decreased with the detuning of the fundamental wavelength from $796 \mathrm{~nm}$ toward the shorter wavelength region. At the same time, a strong enhancement of the $15^{\text {th }}$ harmonic in the case of the $782 \mathrm{~nm}$ radiation was observed, while the intensities of other harmonics remained relatively unchanged. These observations have shown the influence of ionic transitions on the intensity of individual harmonics. In particular, it was demonstrated that the $26 \mathrm{~nm}$ shift of the central wavelength of the main pulse, which corresponded to the $2 \mathrm{~nm}$ shift of the wavelength of $13^{\text {th }}$ harmonic, considerably changed the overall pattern of harmonic distribution at the plateau region.

The tuning of harmonic wavelength by adjustment of the master oscillator is not so easily performed process. As it was mentioned above, a much simpler way for tuning the harmonic wavelength without tuning the laser spectrum can be performed by controlling the chirp of the driving radiation $[15,17]$.

The observed phenomenon of the giant enhancement of single harmonic in the indium plasma has recently been carefully analyzed $[19,21]$. Those studies have shown that the application of shorter pulses ( 35 and $48 \mathrm{fs}$ ) can further improve the output of the $13^{\text {th }}$ harmonic from the indium plasma. The $10^{-4}$ conversion efficiency to the $61 \mathrm{~nm}$ line has 


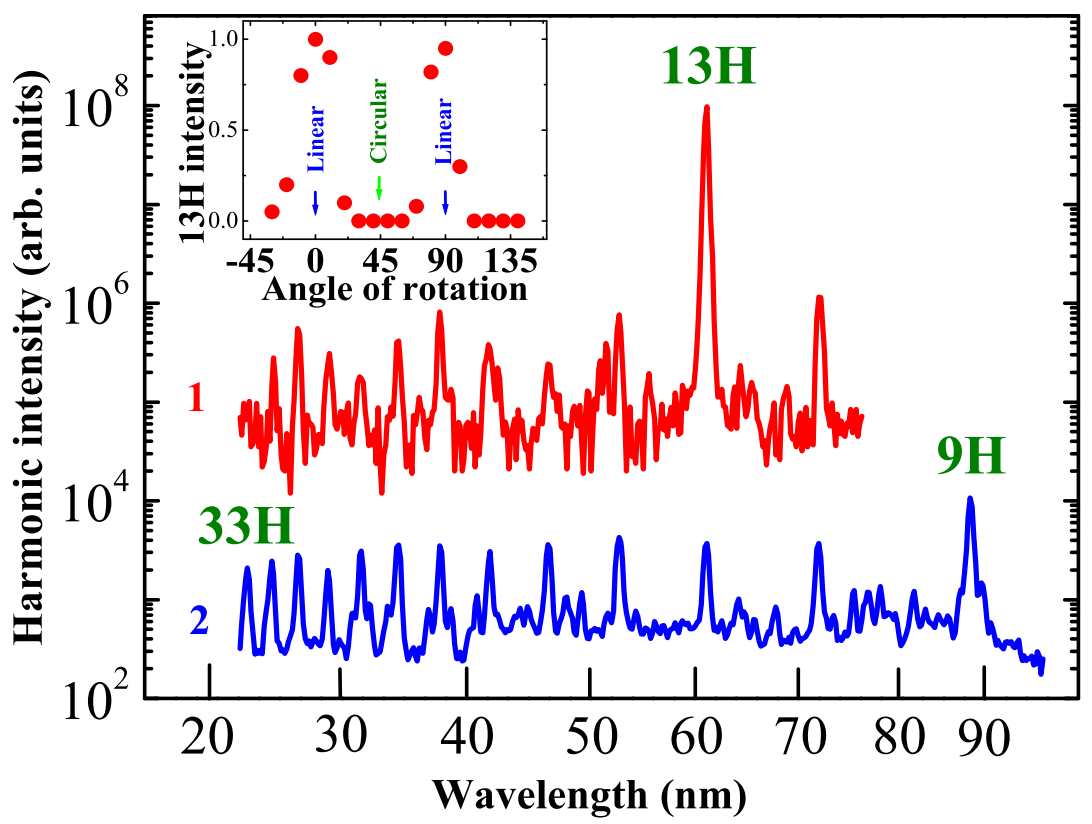

Fig. (2). High-order harmonic spectra obtained in the (1) indium and (2) silver plasma. Curves in this and other figures shifted vertically with respect to each other to avoid overlap for visual clarity. Inset shows the $13^{\text {th }}$ harmonic yield as a function of the rotation of quarterwavelength plate.

been reported, with $200 \times$ enhancement factors for the $13^{\text {th }}$ harmonic with regard to the neighboring ones.

The question arises why one can achieve a strong enhancement of a single harmonic within the harmonic spectrum in the case of indium plasma? A comparison with a past study of the In plasma [22] showed that the emission in the range of $40-65 \mathrm{~nm}$ is due to the radiative transitions to the ground state $\left(4 d^{10} 5 s^{2}{ }^{1} S_{0}\right)$ and low-lying state $\left(4 d^{10} 5 s 5 p\right)$ of In II. Previous work [22] revealed an exceptionally strong line at $62.1 \mathrm{~nm}(19.92 \mathrm{eV})$, corresponding to the $4 d^{10} 5 s^{2}{ }^{1} S_{0}$ $\rightarrow 4 d^{9} 5 s^{2} 5 p\left({ }^{2} D\right){ }^{1} P_{1}$ transition of In II. The oscillator strength $g f$ of this transition has been calculated to be 1.11, which is more than 12 times larger than those of other transitions in this spectral range. This transition can be driven into the resonance with the $13^{\text {th }}$ harmonic $\left(\lambda=61.2 \mathrm{~nm}, E_{\mathrm{ph}}=\right.$ $20.26 \mathrm{eV}$ ) by the AC-Stark shift, thereby resonantly enhancing its intensity. Such intensity enhancement can be attributed to the existence of oscillating electron trajectories that revisit the ionic core twice per laser cycle [10]. Since such trajectories start from the resonantly populated excited state, with a nonzero initial kinetic energy, they still have nonzero instantaneous kinetic energies when they return to the origin. As usual, recombination results in the emission of harmonics, but due to the relatively low probabilities, the population in the laser-driven wave packets increases continuously and the probability for harmonic emission grows with the number of allowed recollisions. This multiple recollision is predicted to enhance harmonics in the spectral ranges close to the atomic and ionic resonances.

\section{SINGLE HARMONIC ENHANCEMENT IN THE Cr, GaAs, AND INSb PLASMAS}

The laser chirp conditions should be chosen properly to suppress the harmonic chirp that broadens the high-order harmonics and reduces their peak intensities. In order to analyze the harmonic variations at different chirps of driving radiation after the optimization of various experimental conditions for the $\mathrm{HHG}$, further studies have been carried out, which were related with the change of the spectral distribution within the driving and harmonic pulses. The chirp of driving laser pulse can be varied by adjusting the separation of the gratings in the pulse compressor. A reduction in the grating separation from the chirp-free condition generates positively chirped pulses, and an increase of the grating separation provides negatively chirped pulses.

HHG from the plasmas of a number of targets was studied prior to choosing the samples, which are suitable for the observation of a single harmonic enhancement in the shortwavelength range. The harmonic distribution from most of plasmas showed a featureless plateau-like shape in the XUV range, while in some cases it demonstrated a steady or even steep decrease of conversion efficiency for each next harmonic order. The harmonics of $48 \mathrm{fs}, 795 \mathrm{~nm}$ radiation generated from the chromium, gallium arsenate, and indium antimonite plasmas were studied in more details due to the observation of abnormal harmonic distribution in the plateau region. These observations were compared with the harmonics generated in the indium plasma.

The harmonics up to the $29^{\text {th }}(27.3 \mathrm{~nm}), 43^{\text {rd }}(18.4 \mathrm{~nm})$, and $47^{\text {th }}(16.9 \mathrm{~nm})$ orders were observed in these experiments with InSb, GaAs, and $\mathrm{Cr}$ plasmas, respectively. These targets showed some enhancement (or decrease) of specific harmonic order. The studies were performed using the chirpfree 48 fs pulses, as well as the chirped pulses. The change of laser chirp resulted in a considerable variation of harmonic distribution in the mid- and end-plateau ranges, due to the tuning of some harmonics toward the ionic transitions [23].

The HHG in Cr plasma showed a considerable variation of the $27^{\text {th }}$ harmonic intensity at different chirps of the driving radiation [24]. At some chirps, the $27^{\text {th }}$ harmonic almost 
disappeared from the harmonic spectrum. At the same time, a strong $29^{\text {th }}$ harmonic $(27.3 \mathrm{~nm})$ was observed in the case of chirp-free pulses. The chirp variation led to a change in the $29^{\text {th }}$ harmonic yield compared with the neighboring harmonics. The maximum ratio of the $29^{\text {th }}$ and $31^{\text {st }}$ harmonic intensities was measured to be 23 . It should be noted that, for negatively chirped pulses, the harmonic lines became sharper. With further growth of negative chirp, the harmonic spectrum was considerably detuned from the resonances causing the absorption of the $27^{\text {th }}$ harmonic and the enhancement of the $29^{\text {th }}$ harmonic. In that case, the intensities of these harmonics became comparable with each other.

Analogous variations of the harmonic distribution at the end- and mid-plateau ranges were observed in the cases of GaAs and InSb plumes [19, 25]. The change of laser chirp resulted in a tuning of the harmonics generating in the GaAs plasma. At the chirp-free case and for negatively chirped pulses, a featureless plateau-like distribution of high-order harmonics with a gradual decrease of harmonic intensity was observed. However, for positively chirped pulses, an enhanced $27^{\text {th }}$ harmonic $(29.4 \mathrm{~nm})$ was appeared. The intensity of this harmonic was six times higher compared with the intensities of neighboring harmonics.

In the case of InSb plume, a strong $21^{\text {st }}$ harmonic (37.8 $\mathrm{nm}$ ) of chirp-free driving radiation was observed. These studies showed an $8 \times$ enhancement of the $21^{\text {st }}$ harmonic compared with the neighboring harmonics. The $21^{\text {st }}$ harmonic intensity varied at different chirps of pump laser. In particular in the case of positively chirped $140 \mathrm{fs}$ pulses, the $21^{\text {st }}$ harmonic exceeded the neighboring ones by a factor of 10. The enhancement of this harmonic considerably decreased in the case of negatively chirped pulses.

The origin of the enhanced emission in the vicinity of $27.3 \mathrm{~nm}$ (the $29^{\text {th }}$ harmonic from the Cr plasma), $29.4 \mathrm{~nm}$ (the $27^{\text {th }}$ harmonic from the GaAs plasma), and $37.8 \mathrm{~nm}$ (the $21^{\text {st }}$ harmonic from the InSb plasma) was analyzed by inserting a quarter-wave plate in the path of the femtosecond laser beam. No harmonics appeared for circularly polarized laser pulses, as expected assuming the nonlinear optical origin of the observed spectra.

The enhancement of the single harmonics belonging to the mid- and end-plateau regions was smaller compared with the enhancement of the $13^{\text {th }}$ harmonic generated in the indium plasma. The enhancement of the $13^{\text {th }}$ harmonic $(200 \times)$ generated in the In plasma, considerably exceeded the enhancements for the $21^{\text {st }}(10 \times), 27^{\text {th }}(6 \times)$, and $29^{\text {th }}(23 \times)$ harmonics generated in the $\mathrm{InSb}, \mathrm{GaAs}$, and $\mathrm{Cr}$ plasmas, respectively. Such a difference was attributed to the different oscillator strengths of the ionic transitions involved in the resonance enhancement of harmonics.

It should be mentioned that the resonant enhancement of single high-order harmonic discussed in the present review has considerably different origin compared with the resonant-like enhancement of the groups of high-order harmonics, which was demonstrated at particular laser intensities [9, $10,26]$. At present, there is no consensus about the physical origin of these resonant-like enhancements. One group of approaches explains them as laser-intensity-induced channel closings, which is related to the long quantum orbits [26]. The second group of explanations suggests that the en- hancement occurs at particular intensities where the atom (ion) is in a state, which is a superposition of the laserdressed ground state and of one or several laser-dressed excited states (or laser-induced states) [10].

It follows from preceding results that the origin of the strong yield of the single harmonics in the plateau region is associated with the resonance-induced growth of nonlinear optical process. Therefore, let us examine the resonanceinduced growth mechanism in a little more detail. Some experimental observations (in particular, the dependences of the harmonic yield on the beam waist position, plasma sizes, and laser radiation intensity) point out the effects related to a collective character of the HHG from laser plasma. Among the factors enhancing harmonic output are the effects related with the difference in the phase conditions for different harmonics. The phase mismatch $\left(\Delta k=n k_{l}-k_{i}\right.$, where $k_{l}$ and $k_{i}$ are the wave numbers of the laser radiation and $i^{\text {th }}$ harmonic) changes due to the ionisation caused by propagation of the driving pulse through the plasma. According to calculations [27], the phase mismatch caused by the influence of free electrons is about one to two orders of magnitude higher than those caused by the influence of atoms and ions. However, at resonance conditions, when the harmonic frequency is close to the frequency of atomic transition, the variation of the wave number of single harmonic could be considerable, and the influence of free-electron-induced mismatch can be compensated by the atomic dispersion for specific harmonic order. In that case, improvement of the phase matching conditions for single-harmonic generation can be achieved. Such a mechanism probably was responsible for the enhancement of above-described nonlinear optical processes in $\mathrm{In}, \mathrm{GaAs}, \mathrm{Cr}$, and InSb plasmas. Analogous single harmonic enhancement has recently been reported in $\mathrm{Sn} \mathrm{[12]} \mathrm{and} \mathrm{Sb} \mathrm{[13]} \mathrm{plasmas,}$ while in the case of Mn plasma, the multiple harmonic enhancements were observed [28].

Recently, the theoretical analysis of resonance-induced enhancement of the HHG in laser plasma was reported [29]. It was shown that the strong intensity enhancement of single harmonic can be explained using the theory presented in [30], generalized to the case of superposition of the states having different parity. The excited state is embedded into the continuum and is metastable. The crucial point of this consideration is that the transition probability between the ground and the excited state is large and that the excitation energy is resonant with integer multiple of the laser photon energy. A small Stark shift of the ionic state levels can be used to drive the system into the exact resonance.

\section{SINGLE HARMONIC ENHANCEMENT AT STRONG EXCITATION CONDITIONS}

In the studies reviewed in previous sections, no significant difference between the sharpness of harmonics in different parts of plateau region was observed in the case of chirp-free laser pulses. No considerable influence of the selfphase modulation (SPM) on the spectral distribution of harmonics was expected, since the experimental conditions (low density plasma, moderate laser intensities) restricted the possibility of the influence of strongly ionized medium on the phase characteristics of harmonics. An over-ionized medium, with the electron density in the central part higher than in the outer region, acts as a negative lens, leading to a defocusing 
of the laser beam in a plasma and hence to a reduction in the effective harmonic generation volume. In addition, the rapidly ionizing high-density medium modifies the temporal structure of the femtosecond laser pulse due to the SPM.

The below reviewed studies of the HHG in silver plasma were carried out to demonstrate the harmonic tuning at variable intensities of the driving radiation, which allowed in some cases achieving the resonance enhancement of single harmonic [23]. The maximum intensity of the femtosecond beam at the focal spot was $4 \times 10^{17} \mathrm{~W} \mathrm{~cm}^{-2}$. This intensity considerably exceeded the barrier suppression intensity for singly charged metal ions. Necessary care was taken to optimize the high-order harmonic output by adjusting the position of the laser focus by placing it in front of the laser plasma or behind it. The intensity of driving laser pulse at the preformed plasma in that case was varied between $1 \times 10^{14}$ and $5 \times 10^{15} \mathrm{~W} \mathrm{~cm}^{-2}$. This intensity was varied by changing the position of focusing lens with respect to the position of plasma. It was shown that, with the approach of the focal spot toward the plasma area, which corresponds to the increase of laser intensity inside the plasma, the harmonics were tuned up to $1.2 \mathrm{~nm}$ (for the $19^{\text {th }}$ harmonic generating in the silver plasma).

Tuning of the harmonic wavelength could be achieved by (a) tuning the fundamental wavelength of laser pulse [14, 31 , (b) chirping the laser radiation [15, 21, 32], (c) altering the laser intensity, which leads to the control of the ionization rate of the nonlinear medium [23,33,34], and (d) adaptive control using the shaped laser pulses [35]. There is also another method of harmonic tuning. In the case of broadband radiation, the second harmonic generation from the nonlinear crystals (BBO, KDP) can be optimized at different phasematching conditions thus allowing the tuning of second harmonic wavelength. This follows by the tuning of the highorder harmonics using second harmonic pulses as the driving radiation.

Note that the effective tuning using the chirping technique and variable second harmonic radiation technique can be achieved only in the case of broadband radiation. In that case, only the leading part of fundamental pulse consisting on either blue or red components participates in the HHG. In the case of narrowband radiation, the difference in the components at the leading and trailing parts of the chirped pulse is negligible. However, the interplay between the SPMinduced chirp and the artificial chirp of narrowband radiation induced by variation of the distance between gratings in compressor can lead to the adjustment of some harmonics toward the quasi-resonance conditions, which in turn can alter the harmonic output. This process has recently been reported during HHG studies in the case of the plasma consisting of the GaN nanoparticles [36].

The GaN powder consisting of $20 \mathrm{~nm}$ nanoparticles was glued on the substrates and then irradiated by the prepulse. Fig. (3) presents a few lineouts of the harmonic spectra at different chirps of the narrowband driving radiation $(10 \mathrm{~nm}$ full widths at half maximum). The harmonic generation was followed by the plasma emission from $\mathrm{GaN}$ nanoparticles in the XUV region, which dominated by the strong 50.3 and $53.8 \mathrm{~nm}$ ionic transitions marked by two lines. In the case of negatively chirped 280 fs pulses, the $15^{\text {th }}$ harmonic wavelength $(52.7 \mathrm{~nm})$ was just between these two ionic transitions (curve 1). The application of chirp-free pulses led to the redshift-induced tuning of $15^{\text {th }}$ harmonic toward the longerwavelength transition (curve 2). This redshift is consistent with previously reported discussion of the role of nanoparticles and clusters in the shift of fundamental radiation wavelength [37]. Simultaneously, both the enhanced $15^{\text {th }}$ harmonic and higher-order harmonics were the main features of this spectral pattern. A decrease of the intensity of chirp-free pulses (by decreasing the pulse energy) led to less influence of the SPM-induced redshift, which caused the detuning of this harmonic toward the blue side, when one can distinguish the ionic transition at the longer-wavelength wing of $15^{\text {th }}$ harmonic spectrum (curve 3). The positive chirp led to further detuning of this harmonic toward the shorter wavelength ionic transition due to the decrease of pulse intensity (curves 4 and 5).

The experiments with GaN nanoparticle-containing plasma revealed the opportunity of the resonance-induced modification of harmonic spectra using the intensity-induced shift of harmonic wavelength. As it was shown in previous section, the resonance-induced enhancement of single harmonic was achieved using the chirping of the broadband laser pulses. This led to the tuning of harmonics toward the strong ionic transitions due to re-distribution of laser spectrum along the main pulse and preferential harmonic generation for the leading part of this pulse [25, 32]. The defocusing properties of plasma weakened trailing part of the pulse. That is why the leading part of chirped pulse was mainly responsible for the harmonic tuning.

The control over tuning through the change of average intensity leading to the variation of ionization state of argonfilled capillary has recently been examined [34]. The harmonic tuning was shown to depend on the nonlinear spectral shift of fundamental laser pulse caused by ionization, rather than directly on the artificial chirp imposed on the fundamental wavelength. In most of resonance-related HHG experiments, the harmonic spectrum was studied as a function of the laser intensity to show the existence of enhancements for particular intensities $[10,38,39]$. This technique considerably distinguishes from the early approach where the variation of the driving radiation spectrum tuned the harmonic wavelength and adjusted it to coincide with the atomic or ionic resonances in the nonlinear medium. The abovediscussed approach is close to the latter one, though no change of the spectrum of the fundamental radiation was imposed, but instead the change of the spectral distribution inside the pulse was carried out by controlling the chirp of laser radiation.

\section{RESONANCE-INDUCED ENHANCEMENT OF THE HARMONICS OF ULTRAVIOLET RADIATION}

The coherence length $\left(L_{\mathrm{c}}=\pi / \Delta k\right)$ for shorter wavelength radiation is expected to be few times longer for the same spectral range, which can be considered as an advantage of using the $400 \mathrm{~nm}$ pump instead of $800 \mathrm{~nm}$ radiation. This allows increasing the plasma length $\left(L_{\mathrm{p}}\right)$ in the former case, thus enhancing the harmonic yield $I_{\mathrm{h}}\left(I_{\mathrm{h}} \propto L_{\mathrm{p}}{ }^{2}\right)$. Some other advantages of shorter wavelength source include higher 


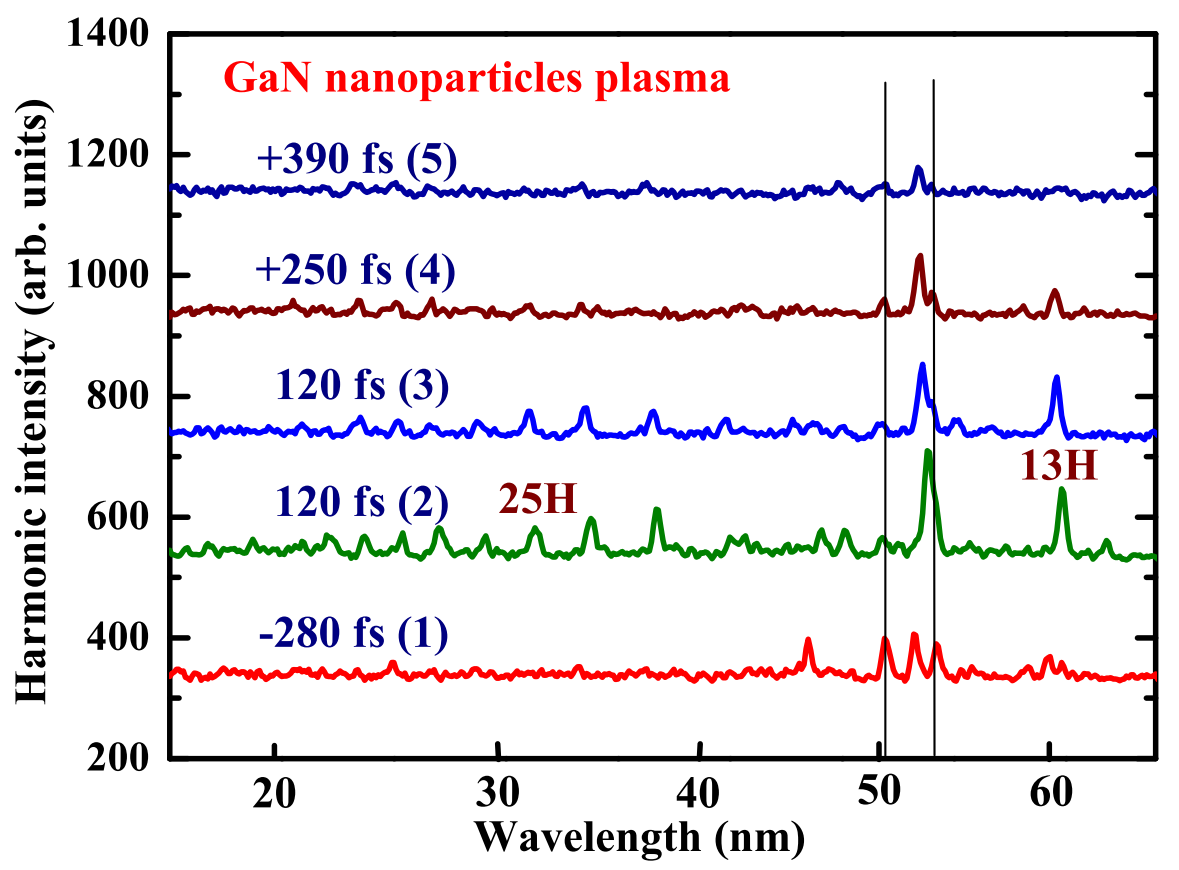

Fig. (3). Harmonic spectra obtained in the GaN nanoparticle-containing plasma using the radiation with variable chirp and pulse duration.

harmonic efficiency at the specific spectral ranges. In particular, low-order harmonics from the shorter wavelength sources correspond to the mid-order harmonic of the $800 \mathrm{~nm}$ pulses. This means that, in the range of $100-200 \mathrm{~nm}$, the harmonic energy from the $400 \mathrm{~nm}$ driving pulses will be higher compared with the harmonics generated by the 800 nm pump.

At $400 \mathrm{~nm}$ pump, some of plasma samples ( $\mathrm{Sn}, \mathrm{Mn}, \mathrm{Cr}$, $\mathrm{Sb})$ demonstrated the resonance-induced enhancement of single harmonic [40]. Such enhancement occurred when the harmonic wavelength was in the vicinity of the radiative transition possessing strong oscillator strength. Fig. (4a) shows the enhanced yield of the $9^{\text {th }}$ harmonic generated in the tin plasma. The intensity of enhanced harmonic eight to ten times exceeded those of the neighboring orders.

Analogous enhancement in this spectral region has previously been reported in the case of harmonic generation in the tin plasma using the fundamental radiation of Ti: sapphire laser [12]. Such an enhancement using the $800 \mathrm{~nm}$ pump has also been observed in the reviewed studies (Fig. 4a). The closeness of the wavelengths of the $17^{\text {th }}$ harmonic of $800 \mathrm{~nm}$ pump (47 nm, $E_{\mathrm{ph}}=26.45 \mathrm{eV}$ ) and the $9^{\text {th }}$ harmonic of 400 nm pump $\left(44.4 \mathrm{~nm}, E_{\mathrm{ph}}=28 \mathrm{eV}\right)$ in the area of strong transitions of singly charged $\mathrm{Sn}$ ions $(25-27 \mathrm{eV})$ showed identical resonance-induced enhancement. The influence of $4 d^{10} 5 s 5 p$ $\rightarrow 4 d^{9} 5 s 5 p^{2}$ transitions $(28.33-28.71 \mathrm{eV})$ of Sn III on the enhancement of the $9^{\text {th }}$ harmonic of $400 \mathrm{~nm}$ radiation could be even stronger compared with the Sn II transitions due to the high $g f$ values of the former transitions.

These HHG studies using the $400 \mathrm{~nm}$ radiation confirmed previously reported peculiarities of harmonic spectra in the vicinity of the $3 p \rightarrow 3 d$ transitions of the $\mathrm{Cr}$ II ions (Fig. 4b). The observed three-fold enhancement of the $15^{\text {th }}$ harmonic of $400 \mathrm{~nm}$ pump can be attributed to the influence of these transitions, though not so pronounced compared with the $29^{\text {th }}$ harmonic of $800 \mathrm{~nm}$ radiation.
In the case of Mn plasma, the maximum harmonic order ( $21^{\text {st }}$ harmonic) was considerably lower compared with the case of $800 \mathrm{~nm}$ pumps (101 ${ }^{\text {st }}$ harmonic). The six-fold enhancement of the $17^{\text {th }}$ harmonic was observed in those studies (Fig. 4c). The single harmonic enhancement in the case of $400 \mathrm{~nm}$ pump was in a stark contrast with the multiple harmonic enhancement observed in the case of $800 \mathrm{~nm}$ driving pulses (Fig. 4c). In the latter case, the harmonics between the $33^{\text {rd }}$ and $41^{\text {st }}$ orders demonstrated the three-fold enhancement compared with the neighboring harmonics.

\section{CONCLUSION}

We reviewed various schemes of the resonance-induced enhancement of the single high-order harmonics generating in the low-ionized laser-produced plasmas. This technique allowed achieving the strong coherent XUV radiation at the specific wavelengths. Initially observed in the low-excited indium plasma, these single harmonic studies have recently been extended to other materials where the strong enhancement of single harmonic was found in the shorter wavelength range. Current state of harmonic efficiencies for single harmonic at the plateau region $\left(10^{-4}\right)$ can also be improved by appropriate choosing the plasma configuration. Recently, some new single-harmonic schemes involving complex targets (i.e. double target schemes [41] and diatomic molecules [42]) were analyzed both experimentally and theoretically.

Summarizing, the newly developed technique allowed for achieving better efficiency for the conversion of laser energy from the well developed visible and near-infrared laser sources toward the short wavelength range.

\section{ACKNOWLEDGEMENTS}

Author thanks H. Kuroda, M. Suzuki, M. Baba, P. D. Gupta, P. A. Naik, and T. Ozaki for fruitful discussions at various stages of collaboration. Author also gratefully acknowledges the support from Japan Society for the Promotion of Science, Natural Sciences and Engineering Research 

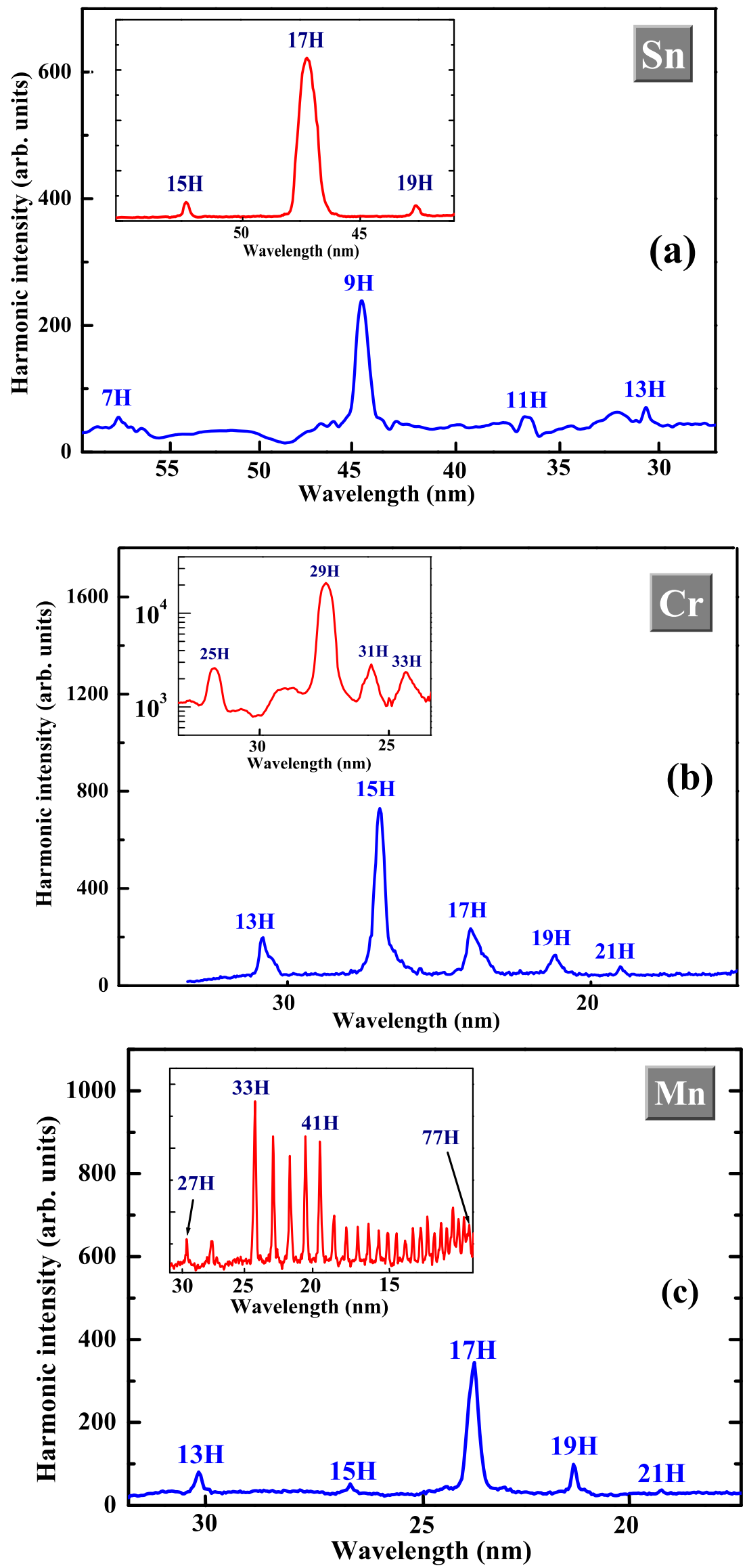

Fig. (4). Single harmonic enhancement observed in the case of the $400 \mathrm{~nm}$ radiation propagating through the (a) tin, (b) chromium, and (c) manganese plasmas. Insets show the enhancement of single and multiple harmonics obtained in the case of $800 \mathrm{~nm}$ pump. 
Council of Canada, and Raja Ramanna Centre for Advanced Technology, India.

\section{REFERENCES}

[1] Chang Z, Rundquist A, Wang H, Murnane MM, Kapteyn HC. Generation of coherent soft X-Rays at $2.7 \mathrm{~nm}$ using high harmonics. Phys Rev Lett 1997; 79: 2967-70.

[2] Gisselbrecht M, Descamps D, Lynga C, L'Huillier A, Walhstrem CG, Meyer M. Absolute photoionization cross sections of excited He states in the near-threshold region. Phys Rev Lett 1999; 82: 4607-10.

[3] Bauer M, Lei C, Read K, et al. Direct observation of surface chemistry using ultrafast soft-X-Ray pulses. Phys Rev Lett 2001; 87: 025501 .

[4] Nagasono M, Suljoti E, Pietzsch A, et al. Resonant two-photon absorption of extreme-ultraviolet free-electron-laser radiation in helium. Phys Rev A 2007; 75: 051406.

[5] Paul PM, Toma ES, Breger P, et al. Observation of a train of attosecond pulses from high harmonic generation. Science 2001; 292: 1689-92.

[6] L'Huillier A, Descamps D, Johansson A, Norin J, Mauritsson J, Wahlström CG. Applications of high-order harmonics. Eur Phys J D 2003; 26: 91-8.

[7] Rundquist A, Durfee CG, Chang ZH, et al. Phase-matched generation of coherent soft X-Rays. Science 1998; 280: 1412-15.

[8] Kazamias S, Douillet D, Weihe F, et al. Global optimization of high harmonic generation. Phys Rev Lett 2003; 90: 193901.

[9] Figueira de Morisson Faria C, Copold R, Becker W, Rost JM. Resonant enhancements of high-order harmonic generation. Phys Rev A 2002; 65: 023404.

[10] Taieb R, Veniard V, Wassaf J, Maquet A. Roles of resonances and recollisions in strong-field atomic phenomena. II. High-order harmonic generation. Phys Rev A 2003; 68: 033403.

[11] Gibson EA, Paul A, Wagner N, et al. Coherent soft X-Ray generation in the water window with quasi-phase matching. Science 2003; 302: 95-8.

[12] Suzuki M, Baba M, Ganeev RA, Kuroda H, Ozaki T. Anomalous enhancement of single high-order harmonic using laser ablation tin plume at $47 \mathrm{~nm}$. Opt Lett 2006; 31: 3306-8.

[13] Suzuki M, Baba M, Kuroda H, Ganeev RA, Ozaki T. Intense exact resonance enhancement of single-high-harmonic from an antimony ion by using Ti:sapphire laser at $37 \mathrm{~nm}$. Opt Express 2007; 15: 1161-6.

[14] Ganeev RA, Suzuki M, Ozaki T, Baba M, Kuroda H. Strong resonance enhancement of a single harmonic generated in extreme ultraviolet range. Opt Lett 2006; 31: 1699-701.

[15] Chang Z, Rundquist A, Wang H, Christov I, Kapteyn HC, Murnane MM. Temporal phase control of soft-X-Ray harmonic emission. Phys Rev A 1998; 58: R30-3.

[16] Kim HT, Lee DG, Hong KH, Kim JH, Choj IW, Nam CH. Continuously tunable high-order harmonics from atoms in an intense femtosecond laser field. Phys Rev A 2003; 67: 051801.

[17] Kim HT, Kim JH, Lee DG, et al. Optimization of high-order harmonic brightness in the space and time domains. Phys Rev A 2004; 69: 031805 .

[18] Ganeev RA, Suzuki M, Baba M, Kuroda H. Generation of strong coherent extreme ultraviolet radiation from the laser plasma produced on the surface of solid targets. Appl Phys B 2005; 81: 10819.

[19] Ganeev RA, Singhal H, Naik PA, et al. Harmonic generation from indium-rich plasmas. Phys Rev A 2006; 74: 063824.

[20] Ganeev RA, Elouga Bom LB, Kieffer JC, Ozaki T. Optimum plasma conditions for the efficient high-order harmonic generation in platinum plasma. J Opt Soc Am B 2007; 24: 1319-23.
[21] Ganeev RA, Elouga Bom LB, Kieffer JC, Ozaki T. Systematic investigation of resonance-induced single harmonic enhancement in the extreme ultraviolet range. Phys Rev A 2007; 75: 063806.

[22] Duffy G, Dunne P. The photoabsorption spectrum of indium laserproduced plasma. J Phys B At Mol Opt Phys 2001; 34: L173-8.

[23] Ganeev RA, Naik PA, Singhal H, Chakera JA, Kuroda H, Gupta PD. Tuning of the high-order harmonics generated from laser plasma plumes and solid surfaces by varying the laser spectrum, chirp, and focal position. J Opt Soc Am B 2007; 24: 1138-43.

[24] Ganeev RA, Suzuki M, Baba M, Kuroda H. Harmonic generation in XUV from chromium plasma. Appl Phys Lett 2005; 86: 131116.

[25] Ganeev RA, Singhal H, Naik PA, et al. Single harmonic enhancement by controlling the chirp of the driving laser pulse during highorder harmonic generation from GaAs plasma. J Opt Soc Am B 2006; 23: 2535-40.

[26] Milošević DB, Becker W. Role of long quantum orbits in highorder harmonic generation. Phys Rev A 2002; 66: 063417.

[27] Kubodera S, Nagata Y, Akiyama Y, et al. High-order harmonic generation in laser-produced ions. Phys Rev A 1993; 48: 4576-81.

[28] Ganeev RA, Elouga Bom LB, Kieffer JC, Suzuki M, Kuroda H, Ozaki T. Demonstration of the 101st harmonic generated from laser-produced manganese plasma. Phys Rev A 2007; 76: 023831.

[29] Milošević DB. High-energy stimulated emission from plasma ablation pumped by resonant high-order harmonic generation. J Phys B At Mol Opt Phys 2007; 40: 3367-76.

[30] Milošević DB. Theoretical analysis of high-order harmonic generation from a coherent superposition of states. J Opt Soc Am B 2006; 23: 308-17.

[31] Shan B, Cavalieri A, Chang Z. Tunable high harmonic generation with an optical parametric amplifier. Appl Phys B 2002; 74: S23-6.

[32] Ganeev RA, Naik PA, Singhal H, Chakera JA, Gupta PD. Strong enhancement and extinction of single harmonic intensity in the mid- and end-plateau regions of the high harmonics generated in low-excited laser plasmas. Opt Lett 2007; 32: 65-7.

[33] Tosa V, Kim HT, Kim IJ, Nam CH. High-order harmonic generation by chirped and self-guided femtosecond laser pulses. II. Timefrequency analysis. Phys Rev A 2005; 71: 063808.

[34] Froud CA, Rogers ETF, Hanna DC, et al. Soft-X-Raywavelength shift induced by ionization effects in a capillary. Opt Lett 2006; 31 : 374-6.

[35] Reitze DH, Kazamias S, Weihe F, et al. Enhancement of high-order harmonic generation at tuned wavelengths through adaptive control. Opt Lett 2004; 29: 86-8.

[36] Ganeev RA, Suzuki M, Redkin PV, Baba M, Kuroda H. Variable pattern of high-order harmonic spectra from laser-produced plasma by using the chirped pulses of narrow-bandwidth radiation. Phys Rev A 2007; 76: 023832 .

[37] Kim KY, Alexeev I, Antonsen TM, Gupta A, Kumarappan V, Milchberg HM. Spectral redshifts in the intense laser-cluster interaction. Phys Rev A 2005; 71: 011201R.

[38] Toma ES, Antoine P, de Bohan A, Muller HG. Resonanceenhanced high-harmonic generation. J Phys B At Mol Opt Phys 1999; 32: 5843-52

[39] Xu H, Tang X, Lambropoulos P. Nonperturbative theory of harmonic generation in helium under a high-intensity laser field: The role of intermediate resonance of the ion. Phys Rev A 1992; 46: R2225-8.

[40] Ganeev RA, Elouga Bom LB, Ozaki T. High-order harmonic generation from plasma plume pumped by 400-nm wavelength laser. Appl Phys Lett 2007; 91: 131104.

[41] Ganeev RA, Milošević DB. Comparative analysis of the high-order harmonic generation in the laser ablation plasmas prepared on the surfaces of complex and atomic targets. J Opt Soc Am B 2008; 25: 1127-34.

[42] Ganeev RA, Redkin PV. Role of resonances in the high-order harmonic enhancement in diatomic molecules. Opt Commun 2008; 281: 4126-9. 